\title{
Transient Tachypnea of Newborn in Neonatal Care Unit of Children Welfare Hospital Medical City /Baghdad
}

\author{
Numan N. Hameed \\ Manal B. Naaom \\ Ali J. Shareef
}

\author{
*MD, FRCPCH, FIBMS, MAAP, MRCPCH, DCH \\ **MD, CABP \\ $* * \mathrm{MD}, \mathrm{MBChB}$
}

J Fac Med Baghdad

2015; Vol.57, No.1

Received Jan. 2015

Accepted Feb.2015

Background: Transient tachypnea (TTN) is a common disorder of the newborn. It is characterized by the early onset of tachypnea sometimes with retractions or expiratory grunting and occasionally cyanosis that is relieved by minimal oxygen supplementation $(<40 \%)$.

Objectives: To identify the risk factors and describe the clinical characteristics, treatment and outcome of infants with TTN.

Patients and methods: This study was carried out on 100 newborn babies with birth weight of 2500 to 4000 gm and gestational ages range from (completed $37-42 \mathrm{wks}$ ), who were admitted to the neonatal care unit of Children Welfare Teaching Hospital, Medical city, Baghdad, from 1st of September 2013 to the 31st of January 2014, and diagnosed as having TTN.

Results: Males represented $68 \%$ and females were $32 \%$, with a male to female ratio of $2.1: 1$. Fifty-three percent aged 38 weeks, compared to $38 \%$ aged $<38$ weeks and $9 \%>38$ weeks, indicating that there was a significant inverse correlation between the incidence of TTN and the gestational age. There was a highly significant inverse correlation between the neonate's weight and the frequency of TTN. Cesarean section done in $80 \%$ compared to $20 \%$ delivered with spontaneous vaginal delivery (SVD) and neonates who were delivered by elective section were more likely to have TTN than those delivered with SVD or emergency section. The history of maternal diabetes in (17\%), a statistically significant association was found between TTN and the maternal Diabetes Mellitus but not with other maternal diseases. Tachypnea and chest retraction were the most frequent clinical manifestations, 93\% and 72\% respectively. Chest X-ray revealed that $71 \%$ had increased pulmonary vascular markings, over aeration in $22 \%$. Out of the 100 cases, $95 \%$ were discharged after they improved without complications, 5\% complicated with Pneumothorax. No death in the studied group.

Conclusions: There was a significant association between TTN and maternal diabetes, mode of delivery especially elective CS, lower gestational age (38 weeks and less), and lower body weight. The most frequent clinical manifestations of TTN are Tachypnea and chest retraction. Increased pulmonary vascular markings and over- aeration are the most frequent radiological manifestations. TTN is a self-limited disease in most of the cases.

Keywords: Transient Tachypnea of Newborn, Neonatal Care Unit, Cesarean section.

\section{Introduction:}

Transient tachypnea is a common physiologic disorder of the newborn. It is characterized by the early onset of tachypnea sometimes with retractions or expiratory grunting and occasionally cyanosis that is relieved by minimal oxygen supplementation $(<40 \%)(1)$. The initial clinical picture is usually completely resolved by 48 to 72 hours, hence the term transient. Hypercapnia and acidosis are uncommon. Distinguishing the disease from RDS and other respiratory disorders (e.g pneumonia) may be difficult, and transient tachypnea is frequently a diagnosis of exclusion; the distinctive features of transient tachypnea are rapid recovery of the infant

*college of medicine/ Children welfare Teaching hospital,/Medical city, Baghdad, Iraq.

**Children welfare Teaching hospital/ Medical city/Baghdad, Iraq.

,E-Mail: numanalhamdani@yahoo.com. and the absence of radiographic findings for RDS (hypoaeration, diffuse reticulo-granular pattern, air bronchogram) and other lung disorders.(1)Transient tachypnea of the newborn (TTN) is the most common cause of respiratory distress in newborns. Although associated with some morbidity, it is generally believed that once TTN resolves, there is no further increased risk for respiratory disease (2).Transient tachypnea results from pulmonary edema secondary to inadequate or delayed clearance of fetal alveolar fluid. Fetal alveolar fluid is continuously secreted during pregnancy through an epithelial chloride secretion mechanism and the rate of secretion decreases a few days before delivery (3). Risk factors for TTN include: Birth by cesarean section with or without labor, Precipitous birth, Preterm birth, Male gender, Multiple gestations, Maternal diseases: Diabetes mellitus and Asthma and Macrosomia.(4) Other obstetric factors such as excessive 
maternal sedation, prolonged labor and volume of maternal intravenous fluid have been less consistent (5).Treatment is mainly supportive with provision of supplemental oxygen as needed. More severe cases may respond to continuous airway pressure (CPAP) to improve lung recruitment. TTN is a selflimited disease with no risk of recurrence and the prognosis is excellent $(3,4)$. This study aimed to identify the risk factors, the clinical characteristics, treatment and outcome of infants with TTN.

\section{Patients and methods:}

A descriptive study was carried out on 100 term neonates (completed $37-42 \mathrm{wk}$ ) who were admitted to the NCU in children welfare hospital in medical city from the 1 st of September 2013 to the 31 st of January 2014, and diagnosed as having Transient Tachypnea of Newborn(TTN).The gestational age was determined using (the first day of the last menstrual period dating by the mother and/or obstetric ultrasonography in the first trimester and Ballard score postnatally). The body weight of the affected neonates ranged $2.5-4 \mathrm{~kg}$. Information regarding gestational age, body weight, clinical manifestations, risk factors as, maternal diseases (D.M, Asthma, Hypertension, and anemia), maternal drug history (dexamethasone), lines of treatment, complications was obtained from medical records and baby care giver. Diagnosis of TTN was made by pediatricians based on history and clinical manifestations (early onset of tachypnea that responds to oxygen within 48-72 hrs, and exclusion of other causes of respiratory distress) and was supported by radiographic findings on C-XR (increased pulmonary vascular markings, over aeration of the lungs). $\mathrm{C}$-XR was done for all neonates and seen by radiologist, who described the radiological findings in a report for each neonate. All neonates were sent for CBP, CRP, and Blood culture. All those who had positive CRP and/or positive Blood culture results were excluded from the study.

Statistical analysis: Data of the 100 neonates were entered and analyzed by using the statistical package for social sciences (SPSS) software for windows version 20. Descriptive statistics were presented as median, ranges, frequencies, (number of cases) and proportions (\%).Cross-tabulation and chi square test were used to assess the significance of differences in frequencies of the variables. Level of significance as $\mathrm{P}$ value of $\leq 0.05$ was considered as significant and $\leq 0.001$ as highly significant, $\mathrm{P}$ value $>0.05$ as not significant. Results and findings were presented in tables and figures.

\section{Results:}

Sixty-Eight percent of patients were males, $32 \%$ were females, with a male to female ratio of $2.1: 1$, so TTN was more frequent among males than females and a highly significant correlation had been found between the incidence of TTN and male gender, $(\mathrm{P}$ value $<0.001)$, (Table 1$)$. The median age of gestational age was 38 weeks and the range was 37 to 40 weeks. According to the value of the median, the studied group was divided into three groups (below, above and the median), this revealed that more than half (53\%) of the studied group aged 38 weeks, compared to $38 \%$ aged less than 38 weeks and $9 \%$ aged $>38$ weeks, indicating that there was a significant inverse correlation between the incidence of TTN and the gestational age, (Table1). There was a highly significant inverse correlation between the neonate's weight and the incidence of TTN, the incidence much decreased with the larger weight, $(\mathrm{P}<0.001)$, (Table 1). Cesarean section was the dominant mode of delivery in $80 \%$ of the cases, compared to $20 \%$ delivered with SVD. Elective cesarean section was the most frequent type of cesarean section, in $72 \%$ vs. only $8 \%$ of emergency cesarean. There was a highly significant correlation between the elective cesarean section and the incidence of TTN. The history of diabetes was positive in mothers of (17\%) of neonates, hypertension in (11\%), Asthma in (9\%), anemia with pregnancy in $(1 \%)$. Statistically significant association had been found between the incidence of TTN and the maternal Diabetes Mellitus but not with other diseases (P.value:0.01). Tachypnea and chest retraction were the most frequent clinical manifestations among studied group; $93 \%$ and $72 \%$, respectively. Expiratory grunting was reported in $45 \%$ and cyanosis in $5 \%$ of the cases. Chest X-ray revealed that (71\%) had increased pulmonary vascular marking, over-aeration in $(22 \%)$ and normal $\mathrm{C}$-XR findings in (7\%). Oxygen mask was used as a line of treatment in (83\%), CPAP in (13\%) and oxygen by nasal cannula in $(4 \%)$. The duration of treatment ranged from 1 to 5 days accordingly, with a median duration of 3 days.Out of the 100 cases, (95\%) were discharged after they improved without complication, 5\% were complicated with pneumothorax, no death in the studied group.There were 13 cases needed CPAP during treatment, among these cases there was statistically significant correlation between CPAP and lower gestational age ( $<38$ weeks), $(\mathrm{P}=0.037)$, lower body weight of neonate $(\mathrm{P}=0.026)$, elective $\mathrm{CS}(\mathrm{P}=0.018)$. Maternal chronic diseases showed no significant association with CPAP $(\mathrm{P}>0.05)($ Table 2).

Correlation of CPAP with gender and pneumothorax: In table 3 , males represented (69.2\%) of the 13 CPAP neonates and (69\%) of 87 neonates on the mask \&nasal cannula, while females represented $30.8 \%$ vs. $31 \%$ respectively, the difference was statistically not significant, $\mathrm{P}>0.05$. Regarding the correlation between pneumothorax and CPAP it had been significantly found that pneumothorax was more frequent with CPAP than mask \&nasal cannula, 3 of the 13 neonate on CPAP (23.1\%) complicated with pneumothorax while out of the 87 neonates on the mask \&nasal cannula, only $(2.3 \%)$ were complicated with pneumothorax, $\mathrm{P}=0.01$ (Table 3 ).

Table1: Demographic characteristics of 100 neonates

\begin{tabular}{cccc}
\hline Variables & number & percent & P value \\
\hline Gender & & & \\
Male & 68 & 68.0 & $<0.001 \quad$ Highly \\
Female & 32 & 32.0 & significant \\
\hline
\end{tabular}




\begin{tabular}{cccc}
\hline $\begin{array}{c}\text { Gestational } \\
\text { age }(\mathrm{Wks})\end{array}$ & & & \\
$<38$ & 38 & 38.0 & \\
38 & 53 & 53.0 & $<0.001$ \\
$>38$ & 9 & 9.0 & Highly significant \\
Median & 38 & & \\
Range & $34-40$ & & \\
\hline Weight (Kg) & & & \\
& & & \\
$2.5-3.0$ & 63 & 63.0 & Highly significant \\
$>3-3.5$ & 27 & 27.0 & \\
$>3.5$ & 10 & 10.0 & \\
Median & 3 & & \\
Range & $2.5-4$ & & \\
\hline
\end{tabular}

Table 2: Correlation between CPAP and other variables $(\mathbf{N}=13)$

\begin{tabular}{cccc} 
& & & \\
CPAP & O2mask, & \\
Variables & cannula & P value \\
& percent & No. & \\
& percent & \\
\hline
\end{tabular}

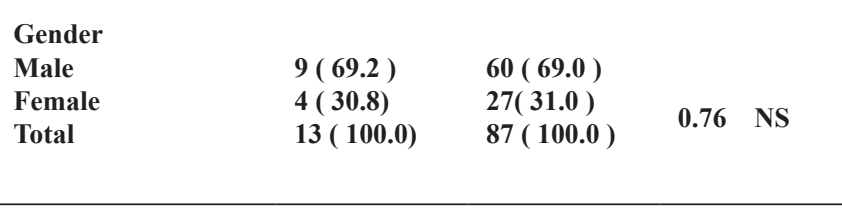

\begin{tabular}{llll} 
Complications & & & \\
& & & \\
Pneumothorax & $3(23.1)$ & $2(2.3)$ & 0.01 Sign. \\
None & $10(76.9)$ & $85(97.7)$ & \\
Total & $13(100.0)$ & $87(100.0)$ & \\
\hline
\end{tabular}

Table 3: Correlation of mode of treatment with gender and pneumothorax

\begin{tabular}{llll}
\hline \multicolumn{1}{c}{ Variables } & $\begin{array}{c}\text { CPAP } \\
\text { Number }\end{array}$ & Percent & P Value \\
\hline Gestational age & & & \\
$<38$ & 9 & 69.2 & \\
38 & 4 & 30.8 & 0.037 \\
$>38$ & 0 & 0.0 & \\
\hline
\end{tabular}

\begin{tabular}{llll} 
Weight & & & \\
$2.5-3.0$ & 10 & 76.9 & \\
$>3.0-3.5$ & 3 & 23.1 & 0.026 \\
$>3.5$ & 0 & 0.0 & \\
& & & \\
\hline Mode of Delivery & & & \\
$\quad$ Elective & 9 & 69.2 & \\
Emergency & 3 & 23.1 & 0.018 \\
SVD & 1 & 7.7 & \\
\hline
\end{tabular}


Demissie K .et al (1998)(13) and Subramanian K N S et al (2006)(14) .there may be a need for larger study to establish this association. Tachypnea and chest retraction were the more frequent clinical manifestations among studied group. Expiratory grunting was less frequent, and the least frequent clinical manifestation was cyanosis.(3)

Radiological findings revealed that the most frequent finding is accentuated pulmonary vascular markings followed by over aeration.(3) The clinical features usually resolved in the first 72 hours. This is compitable with the facts that were documented in pediatric and neonatal textbooks $(1,3)$. Eighty seven percent of the affected neonates received oxygen either by mask $(83 \%)$ or by nasal cannula $(4 \%)$, while $13 \%$ received oxygen by CPAP. Among these cases there was statistically significant correlation between CPAP use and $<38$ weeks gestational age, lower body weight of neonate, elective CS, and male gender. This is in agreement with Tutdibi E, et al and Reuter S et al $(12,15)$ who found that elective cesarean section, lower GA, male gender, and lower birth weight were associated with TTN, and associated with more severe form.Out of the 100 cases, (95\%) were discharged after they had improved without complications, $5 \%$ were complicated with pneumothorax , and it is found that pneumothorax was more frequent with CPAP rather than other lines of treatment (3). Although TTN is a self - limited process, supportive therapy may be accompanied by complications .CPAP is associated with increased risk of air leak (16). Late-preterm delivery, lower initial umbilical artery $\mathrm{pH}(<7.25)$, and lower Apgar score at 1 min were independently associated with poor prognostic treatment outcomes in infants with TTN (17). Conclusions: Males were affected more than females. There was a significant association between TTN and maternal diabetes, mode of delivery especially elective CS, lower gestational age (38 weeks and less), and lower body weight. The most frequent clinical manifestations of TTN are Tachypnea and chest retraction. Increased pulmonary vascular markings and over- aeration are the most frequent radiological manifestations. TTN is a self-limited disease in most of the cases. We recommend delaying elective caesarean section until 39- 40 weeks or until spontaneous labor starts.

\section{Author's contributions:}

NNH participated in the study design, sequence alignment and drafting and finalization of the manuscript. MBN participated in the study design, sequence alignment and finalization of the manuscript. AJS participated in the design of the study, collection of data and performed the statistical analysis. All authors read and approved the final manuscript.

\section{References: \\ 1. Liem JJ, Huq SI, Ekuma O, et al: TranseintTachypniaOf Newborn: Behrman RE, Kliegman RM. Nelson Textbook of Pediatrics 19th ed. 2011; 151:29-33. \\ 2. Cakan M, Nalbantoğlu B, Nalbantoğlu A, Demirsoy U, Say A. Correlation between transient tachypnea of the newborn and wheezing attack. Pediatr Int. 2011 Dec; 53(6):1045-50.}

3. Martin R.J, Fanaroff A.A, Walsh M.C, Neonatal-Perinatal Medicine, 9th Edition, Volume 2, 2012, 1162-1163.

4. Stoll B J, Kliegman RM. Respiratory tract disorder. Behrman RE, Kliegman RM, Jenson HB, eds. Nelson Textbook of pediatrics, 19thed., Philadelphia, Pa: WB Saunders Co;2011:573-588.

5. Hermansen CL, Lorah KN. Respiratory distress in the newborn. American Family Physician 2007; 76(7):987-994.

6. Dani C, Reali MF, Bertini G, Wiechmann L, Spagnolo A,Tangucci $M$, et al. Risk factors for the development of respiratory distress syndrome and transient tachypnea in newborn infants. Eur Respir J 1999;14: 155-159.

7. Asengo M. Transient tachypnea of newborn. Acta Obstet Gynecol Scand. 2007:1-6.

8. Aslan E, Tutdibi E, Martens S, Han Y, Monz D, Gortner L. Transient tachypnea of the newborn (TTN): a role for polymorphisms in the beta- adrenergic receptor (ADRB) encoding genes. Acta Paediatr. 2008 Oct; 97(10):1324-6

9. Levine EM, Ghai V, Barton JJ, Strom CM. M ode of delivery and risk of respiratory diseases in newborns. Clin Obstet Gynecol 2001;97; 439-442.

10. Riskin A, Abend-Weinger M, Riskin-Mashiah S, Kugelman A, Bader D. Cesarean section, gestational age, and transient tachypnea of the newborn. Am J Perinatol. 2005 Oct; 22(7):377-82.

11. Hansen AK, Wisborg K, Uldbjerg N, Henriksen TB. Risk of respiratory morbidity in term infants delivered by elective caesarean section: cohort study. BMJ 2008; 336:85-87.

12. Tutdibi E, Gries K, Bucheler M, Misselwits B, Schlosser $R$, Gortner L. Impact of labor on outcomes in Transient Tachypnea Of The Newborn. Pediatrics 2010 Mar; 125(3): e577-e583.

13. Demissie $K$, Marcella $S W$, Breckenridge $M$ B, Rhoads $G G$. Maternal asthma and transient tachypnea of the newborn .Arch Dis Child 1998; 102:84- 90.

14. Subramanian KNS, BahriM, Kicklighter DS. Transient tachypnea of the Newborn. Pediatr Int.2006; 54:1-11

15. Reuter S, Moser C, Baack M. Respiratory Distress in the Newborn. Pediatrics in Review 2014; 35; 417

16. Cloherty JP, Eichenwald EC, Hansen AR, Stark AR .Transient Tachypnea Of Newborn, in Manual Of Neonatal Care, Seventh edition. 2012; pp 404-406.

17. Seon-YeongBak, Youn-Ho Shin, Ji-Hyun Jeon, Kyu-Hee Park, Jin-Hee Kang, Dong-Hyun Cha, et al.Prognostic factors for treatment outcomes in transient tachypnea of the newborn. Pediatrics International2012; 54: 875-880. IVSL 